\title{
Home based cognitive retraining for cognitive impairments followed by stroke. A case study
}

\author{
Neelam Verma ${ }^{1,}$, Aldrin Anthony Dung Dung ${ }^{2}$, Meena Sharma ${ }^{3}$, Akhila Kumar Panda ${ }^{2}$ \\ ${ }^{1}$ Clinical Psychologist at Nur Manzil Psychiatric Centre, Lal Bagh, Lucknow, India \\ ${ }^{2}$ Department of Neurology, Institute of Human Behaviour and Allied Sciences (IHBAS), Delhi \\ ${ }^{3}$ Department of Clinical Psychology, Institute of Human Behaviour and Allied Sciences (IHBAS), Delhi
}

Email address:

neelampsycho.86@gmail.com (N. Verma), aldrinin@yahoo.com(A. A. D. Dung), meenasharmacp@gmail.com (M. Sharma), drakhilapanda@gmail.com (A. K. Panda)

\section{To cite this article:}

Neelam Verma, Aldrin Anthony Dung Dung, Meena Sharma, Akhila Kumar Panda. Home Based Cognitive Retraining for Cognitive Impairments Followed by Stroke. A Case Study. American Journal of Internal Medicine. Vol. 1, No. 4, 2013, pp. 27-30.

doi: 10.11648/j.ajim.20130104.11

\begin{abstract}
Cognitive difficulties frequently reported in the sufferers of stroke. Limited literatures are available regarding effectiveness of cognitive rehabilitation in post stroke dementia in Indian culture. Aims and objective: To examine the efficacy of home based cognitive retraining (HCR) in a patient who suffered from stroke. Method: Single case study method was opted regarding the same and results of pre and post assessment using NIMHANS neuropsychological battery and MRI of brain were compared. Six month's package of home based cognitive retraining was given to the patient. Results: Pre assessment condition were indicative of fronto-temporal involvement and post assessment revealed significant improvement in cognitive functions i.e. speed of processing, attention, memory and executive function that improve patient's academic performance and daily functioning. Further findings of the present study need validation in discrete settings. Conclusion: Home based cognitive retraining (HCR) is a promising home based technique for treating cognitive impairments caused by stroke.
\end{abstract}

Keywords: Stroke, Cognitive Impairment, Cognitive Retraining

\section{Introduction}

Cognitive difficulties are usually reported in patients with stroke. Attention, memory, language, visuo-spatial skills, executive functions were most affected by stroke [1]. Cognitive rehabilitation further improves person's ability to perform daily activities. Distinct approaches have utilized to amend cognitive impairments after stroke [2, 3]. Researchers followed the basis of neural plasticity which includes the approaches like compensatory, restorative, visuo-spatial and language based therapies, even domain specific retraining were also provided to stroke cases $[4,5]$. However, many stroke related cases do not have the excess of domain specific rehabilitation program, thereby lacking functional recovery that could be due to limited financial resources, transportation difficulties, lack of family support and so forth $[6,7]$.

Home based cognitive retraining provided to patient with stroke has an effective measure which included the rehabilitation of attention, memory and executive functions.
The present case was studied showing effectiveness of the home based rehabilitation procedure in post stroke dementia.

\section{Case Report}

An 18-years old boy pursuing engineering course, suffered from arteriovenous malformation (AVM) that led to left thalamic bleed with mass effect with right sided hemiplegia and non fluent aphasia (Figure 1 and 2). He was surgically treated and improvement in Glasgow coma scale (GCS) score was noted after one week. After two weeks of hospital stay his neurological symptoms recovered to such an extent that he started doing all his daily activities independently. Significant improvement of speech and motor functions were noticed with speech therapy and physiotherapy in next 4 months. During his rehabilitation program, he was also referred for neuropsychological assessment (NPA) and rehabilitation with the complaints of forgetfulness, difficulty in sustaining attention, planning, decision making, mild difficulty in speaking. Patient and his parent's major concern 
was his academic deterioration due to the cognitive deficits.

\section{Neuropsychological Assessment}

Assessment was initiated after detailed review of history. Adequate information was given to the patient and his caregivers regarding the assessment and rehabilitation [8]. Assessment was done using NIMHANS neuropsychological battery which is a comprehensive assessment tool to assess neuropsychological functioning and areas of cognitive deficits. Comprehensive approach was adopted to gather adequate information about the neurocognitive deficits, as parents major concern was patient's academic deteoriation. The battery assesses neuropsychological functioning in the following domains: motor speed, attention, executive functions, comprehension, learning and memory. Assessment was planned to be done in two phases i.e. pre and post assessment phase. Pre assessment results revealed involvement of frontal \& temporal lobe. Following the assessment findings and after identifying the areas of cognitive deficits, home based cognitive retraining was started. Patient's mother was selected to impart rehabilitation as she was found to be more concerned and motivated, even considering her age and educational background i.e., 47 yrs and Bachelor of Ayurvedic and Medical Surgery (BAMS), neuropsychologist decided to take help of mother and asked her to monitor the cognitive retaining program at home. Moreover, mother deliberately left her job during the treatment and devoted full time to the patient. The therapy module comprised six months package of cognitive retraining including psycho-education of family members. Initially, patient was called thrice a week for one month, twice a week in the second month and subsequently once in each month.

\section{Results}

Norms of the NIMHANS neuropsychological battery has been standardized on 540 normal Indian volunteers. Norms are available for different population based on age, gender, and level of education. After obtaining the scores of each test, interpretation of scores was done by comparing the scores of the patient with normal controls. Findings of pre $\&$ post assessment revealed significant improvement of all modalities of cognitive domain after cognitive rehabilitations (Table 1). The graphical representations of pre \& post assessment results were shown in Table 2.

\section{Table 1}

Showing percentile (P) scores of pre \& post assessment condition of cognitive training with significant improvement of different cognitive domain scores. There is significant improvement of various cognitive domains after cognitive retraining.

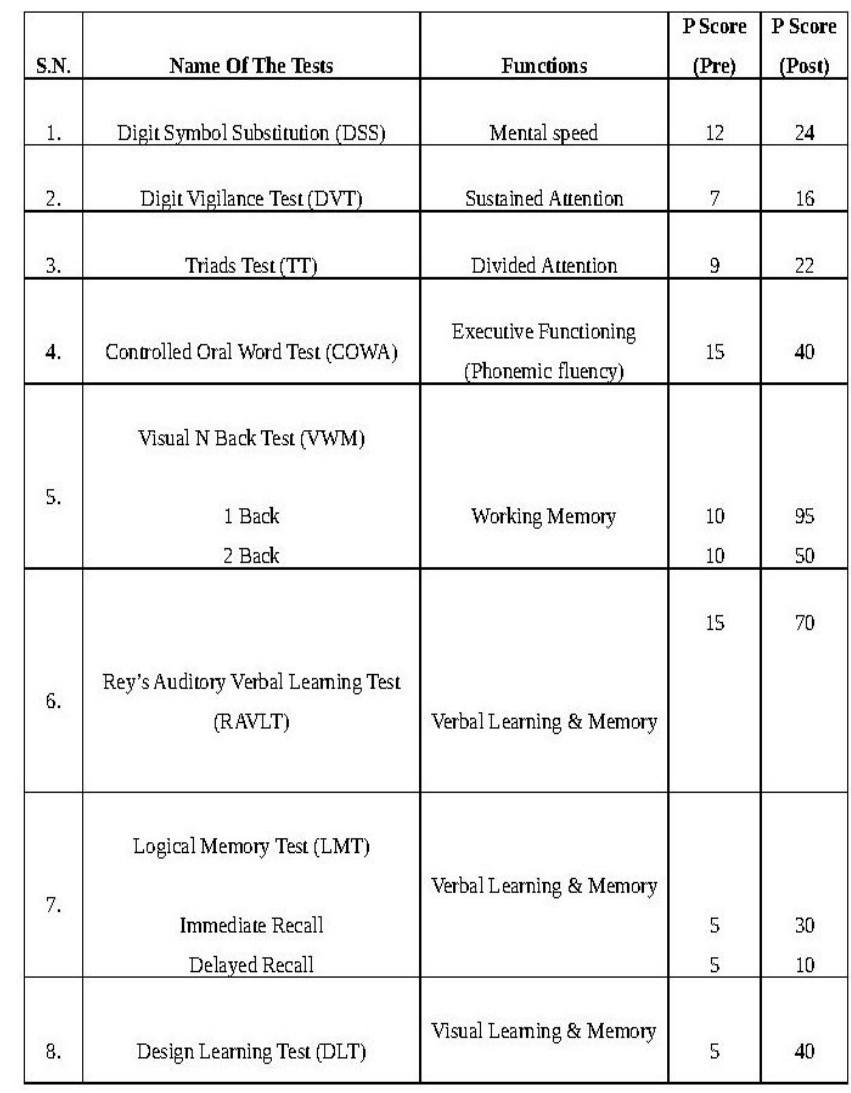

(DSS-Digit Symbol Substitution, DVT- Digit Vigilance Test, TT-Triads Test, COWA-Controlled Oral Word Test, VWM-Visual N Back Test, RAVLT-Rey's Auditory Verbal Learning Test, LMT-Logical Memory Test, DLT-Design Learning Test.

\section{Table 2}

The graphical representation of pre (blue line) \& post assessment (red line) results showing improvement of different cognitive domain after cognitive retraining.

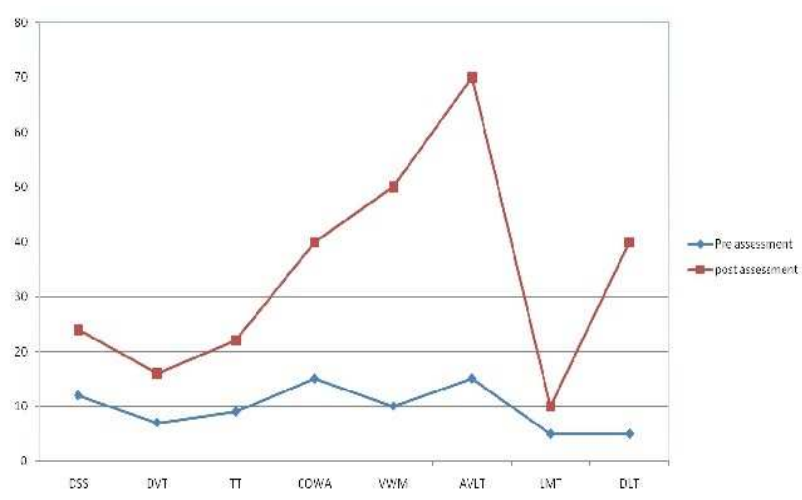

(DSS-Digit Symbol Substitution, DVT- Digit Vigilance Test, TT-Triads Test, COWA-Controlled Oral Word Test, VWM-Visual N Back Test, RAVLT-Rey's Auditory Verbal Learning Test, LMT-Logical Memory Test, DLT-Design Learning Test. 


\section{Discussion}

Stroke is a neurological disorder characterized by cortical and sub cortical deficits represented via imaging techniques and neuropsychological assessment. Substantial literature depicted the executive functions after stroke. In terms of stroke rehabilitation, various approaches utilized to improve the cognitive deficits of persons endured stroke, even home based training program were also formulated to provide rehabilitation services to those sufferers who cannot afford or do not have access to the treatment. In the present study, neuropsychological assessment findings suggested significant recovery in the cognitive domains of processing speed, attention, memory (verbal and visual) and executive functions (Table 1). Existing literatures revealed the importance of home based training program in improving the cognitive deficits of stroke cases. Improvement in speed of processing and attention were noticed using the restoration approach which includes the techniques like practice and rehearsal, spaced retrieval and over learning techniques. In the present case, patient was suggested to develop the practice of reading newspaper daily with loud voice, patient was also advised to indulge himself in outdoor activities such as cycling, playing cricket which enhances his self-confidence. Additionally, patient was asked to practice speaking slowly and clearly at home with his mother tongue and continued this practice daily for faster improvement without setting any limit. At the same time, mother was informed for spaced retrieval where she would be asking the learned information in time intervals from the patient in terms of daily routine and academics. She would also monitor, motivate and gave feedback to the patient for activities at home. In support, one of the studies, adopted similar restorative approach to deal with the stroke cases and found significant effect on performance speed [10]. Although, previous studies made attempt to restore the impaired functions using distinguish strategies, but considering the Indian culture simple and accessible techniques were employed, so patient can have access to it.

On the other hand, another compensatory approach was followed to recover the memory functions. Environmental aids such as diaries, notebook, electronic gadgets, mobiles and alarm watches were recommended to the patient. For instance in the present case patient was given preference of selecting any of the electronic gadgets that would assist in recalling / remembering the assigned task. In support, many studies have followed this approach to improve the cognitive deficit of brain injury cases; this approach was found to be effective in recovering the memory deficits [10].

However, compensatory strategies proved to be effective and also internally generated by the patients on their own, but following this technique over time is quite difficult, therefore in the present case patient was motivated frequently by the mother to improve the deficit areas, intact cognitive functions would be utilized.In addition, findings have also presented the changes in executive function such as planning. Decision making by following both compensatory and restoration approach. Patient was provided responsibilities at home where he was required to plan and organize the task on his own; mother was suggested by the neuropsychologist to assist the patient in using compensatory strategies while planning the task in academics. In support, one more study showed the role of compensatory strategies in improving the disturbances of executive functions [10]. However, patient received adequate stimulation at home to improve his executive functioning but rare efforts have been made by the neuropsychologist to assess the level of participation made by the patient at home, as recent study displayed the improvement of executive functions with the level of patient participation in rehabilitation in patient setting. In the third month of retraining, patient started showing improvement in memory functions followed by improvement in attention. Thereafter, the improvement was significant in all the functions that were targeted. This improvement is corroborated with the results of neuropsychological assessment in post HCR condition and report given by patient's mother about daily activities and academic functioning. Further findings were also confirmed with the MRI report where patient showed significant recovery (Figure 3).

\section{Figures}

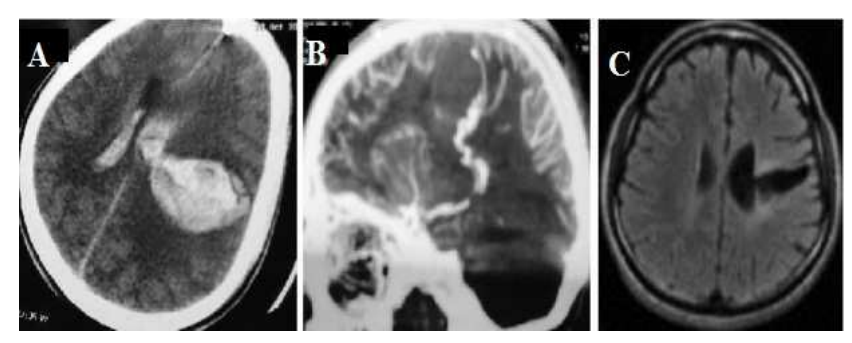

Figure 3. (A) Non contrast computed tomography showing left thalamic hematoma with intraventricular extension, (B) - Sagittal maximum intensity projection (MIP) image, and showing dilated tortuous arteriovenuos malformation. (C)-MRI of brain with T2 sequence showed marked resolution of hematoma volume after 6 month of initial symptoms.

\section{Conclusion}

Home based cognitive retraining (HCR) is a promising home based technique for treating cognitive impairments caused by stroke. This technique is also affordable to persons who are affected with other reversible demented illness. It further improves the day-to-day functioning and academic difficulties. Further studies are required to assess the validity of home-based technique in other settings.

\section{Key Points}

- Home based cognitive retraining (HCR) is a promising home based technique for treating cognitive impairments caused by stroke and other reversible demented illness.

- Rehabilitation approaches like restoration, 
compensation and substitutions were utilized which included the tasks specific to the domains.

- Early neuro- rehabilitation like physiotherapy, speech therapy along with neuro psycho logic assessment with cognitive behaviour therapy will improve the clinical as well as social and functional outcomes in patients of stroke.

\section{References}

[1] Skidmore ER, Whyte EM, Holm MB, Becker JT, Butters MA, Dew MA, Munin MC, Lenze EJ. Cognitive and affective predictors of rehabilitation participation after stroke. Arch Phys Med Rehabil 2010; 91: 203-7.

[2] Sachdev PS, Brodaty H, Valenzuela MJ, Lorentz L, Looi JC, Wen W, Zagami AS. The neuropsychological profile of vascular cognitive impairment in stroke and TIA patients. Neurology 2004; 62: 912-9.

[3] Tatemichi TK, Paik M, Bagiella E, Desmond DW, Pirro M, Hanzawa LK. Dementia after stroke is a predictor of long-term survival. Stroke 1994; 25: 1915-9.

[4] Fish J, Manly T, Emslie H, Evans JJ, et al. Compensatory strategies for acquired disorders of memory and planning: differential effects of a paging system for patients with brain injury of traumatic versus cerebrovascular aetiology. Journal of Neurology, Neurosurgery and Psychiatry 2008; 79(8):930-5.

[5] Ryan J.D., Polatajko H.J., McEwen S et al. Analysis of Cognitive Environmental Support (ACES): preliminary testing. Neuropsychol Rehabil 2011; 21: 401-27.

[6] Schwamm L H, Audebert H J, Amarenco P et al. Recommendations for The implementation of telemedicine within stroke systems of care: a Policy statement from the American Heart Association. Stroke 2009; 40:2635-60

[7] CraiK, F.I. M., \& Watkins, M.J. The role of rehearsal in short-term memory. Journal of Verbal Learning and Verbal Behavior.1973; 12: 599-607.

[8] Rao, Shobini L., D. K. Subbakrishna, and K. Gopukumar. NIMHANS Neuropsychology Battery Manual. National Institute of Mental Health and Neurosciences, 2004

[9] Winkens I, Van Heugten CM, Wade DT, Habets EJ, Fasotti L. Efficacy of time pressure management in stroke patients with slowed information processing: a randomized controlled trial. Arch Phys Med Rehabil. 2009; 90(10):1672-9.

[10] Shallice, T, Burgess P. Deficits in strategy applications following frontal lobe damage in man. Brain 1991; 114: $727-741$ 